INVESTIGACIÓN/RESEARCH

Recibido:01/10/2014---Aceptado: 28/11/2014---Publicado: 15/12/2014

\title{
LA ENSEÑANZA DEL IDIOMA INGLÉS A TEMPRANA EDAD: SU IMPACTO EN EL APRENDIZAJE DE LOS ESTUDIANTES DE ESCUELAS PÚBLICAS
}

David Quidel Cumilaf: Universidad Católica de Temuco. Chile. davidquidel1@gmail.com.

Juan del Valle Rojas: Universidad Católica de Temuco. Chile. dvalle1985@hotmail.com.

Lissette Arévalo López: Universidad Católica de Temuco. Chile.

lisettearevalo23@gmail.com

Catherine Nancucheo Chihuaicura: Universidad Católica de Temuco. Chile. cnancucheo2008@alu.uct.cl

Ramón Ortiz Neira: Universidad Católica de Temuco. Chile.

rortiz86@hotmail.com.

\section{RESUMEN}

La presente investigación aborda la temática de la enseñanza del idioma inglés a temprana edad, cuyo objetivo es develar el impacto de la enseñanza de este idioma como segunda lengua dentro de los primeros años de escolaridad. Los participantes seleccionados fueron estudiantes de enseñanza primaria pertenecientes a cuatro diferentes establecimientos públicos que presentan altos índices de vulnerabilidad. La metodología empleada es de tipo cualitativa, ya que permite mayor flexibilidad y cercanía con la realidad al momento de analizar a los participantes. El plan de análisis se enmarca en el análisis de contenido, de tipo descriptivo. Este permitió hacer inferencias y aplicarlas al contexto en el cual se desarrolló esta investigación. Se desprende como principal hallazgo que los estudiantes que comienzan la exposición del idioma inglés a temprana edad, de manera formal, logran un mejor aprendizaje, comprensión y motivación con el mismo, comprado con aquellos alumnos que comenzaron su proceso de aprendizaje a una edad tardía.

\section{PALABRAS CLAVE}

Inglés - Temprana edad - Comprensión Lectora - Comprensión Auditiva Producción escrita - KET Test - Exposición al Inglés - Comprensión - Motivación. 


\title{
THE ENGLISH LANGUAGE TEACHING AT AN EARLY AGE: THE IMPACT OF THE LEARNING PROCESS IN STUDENTS FROM PUBLIC SCHOOLS
}

\begin{abstract}
This research is about learning English as a second language at an early stage, where its main purpose is to reveal the impact on learning a second language during childhood. The participants were primary level students from four different public schools, which have high rates of vulnerability. The methodology is qualitative and this allows a greater flexibility and closeness within the everyday life context of the participants. The analysis used was the descriptive content analysis. This allowed for the making of inferences and applying them to the context in which this research was developed. Concerning to the main finding is clear that students who began English language exposure at younger ages, formally, achieve better learning, comprehension and motivation to it, than those who started at a later age.
\end{abstract}

\section{KEY WORDS}

English - Early age - Reading comprehension - Listening comprehension - Writing production - KET Test - Language exposure - Comprehension - Motivation.

\section{INTRODUCCIÓN}

Actualmente, en Chile la enseñanza formal del inglés como segunda lengua ha provocado una gran preocupación, tanto del gobierno como de las reformas educativas y docentes que incentivan a los estudiantes al aprendizaje de este idioma. A pesar del avance en las reformas educativas como el Programa Inglés Abre Puertas, creado en el año 2003, que fomentan el desarrollo del inglés como segunda lengua en los alumnos y el avance en la Cobertura Educativa, existen aún muchas interrogantes sobre cuál es la edad óptima para comenzar a enseñar un segundo idioma. En cuanto a la incorporación de la enseñanza del idioma inglés, muchos establecimientos han optado por implementarlo desde primer ciclo de enseñanza básica, mientras que otros comienzan desde quinto año básico hacia adelante. Respecto a establecimientos públicos, privados y subvencionados, no existen en la actualidad muchas investigaciones relacionadas al tema en cuestión. Es en este contexto que las políticas educativas sugieren y plantean distintas reformas para nuestra educación, unos encauzados en un aprendizaje formal que incluya la enseñanza básica, desde kínder, y otra en la que la enseñanza se direccione 
plenamente desde segundo ciclo de enseñanza básica en adelante.

Existen establecimientos que optan por la enseñanza del inglés a temprana edad. Actualmente, se consideran dos teorías que demuestran y avalan la importancia del aprendizaje del inglés a una temprana edad. La primera, se sustenta en que la educación formal del inglés requiere necesariamente de una etapa cognitiva temprana para su eficiente desarrollo, y otra en la que es necesario un conocimiento más estructurado de la lengua materna para poder cimentar las bases. En este contexto se encuentra actualmente la reforma educacional en Chile, estableciendo nuevas formas de direccionar la enseñanza formal del inglés como segunda lengua, puesto que es una necesidad como país el dotar de herramientas que determinen la educación como prioridad y no como un elemento aislado.

De esta forma, se constituye el eje de esta investigación: ¿Cuál es el efecto de los estudiantes que comienzan la enseñanza del inglés a temprana edad? Y ¿De qué forma se ve afectado el rendimiento del inglés en relación a la edad del educando?

Esta investigación tiene como objetivo el aportar a la comprensión sobre la interrogante del periodo crítico y del efecto para la enseñanza de los educandos. Se pretende dar respuestas a las siguientes interrogantes que se relacionan con las mejoras en la educación formal del idioma inglés: ¿Cuál es la implicancia de la edad en relación al aprendizaje de una segunda lengua?, ¿El grado de exposición a una lengua influye realmente en la enseñanza de ésta?, ¿Es la infancia el período más apropiado para aprender una segunda lengua?

Tomando en cuenta lo señalado anteriormente, el problema de investigación se plantea como "Impacto de la enseñanza del Inglés a temprana edad en estudiantes de escuelas públicas de la ciudad de Temuco". Respecto a los conceptos que se abordan en la investigación, se destaca lo siguiente:

\section{Exposición a material auténtico.}

Al considerar el material autentico, nos referimos no sólo a la literatura o textos orales, sino que a la naturaleza del lenguaje expresada por hablantes nativos. Desde esta perspectiva, Geddes y White (1978) recomiendan el uso del material auténtico tanto como sea posible en la enseñanza. El uso de material auténtico debe darse aun cuando parezca que los materiales "inéditos" y "no pedagógicos" puedan crear más problemas de los que sean posibles resolver, dado que los materiales son frecuentemente difíciles de seleccionar, obtener o ser comprendidos por los estudiantes de niveles bajos. 


\section{Exposición al idioma Inglés.}

La exposición al lenguaje dentro de este análisis, corresponde netamente a la interacción a la que pueden haber sido expuestos los alumnos, (estudiantes de una segunda lengua), con hablantes nativos de la lengua inglesa. Long (1996) "Existen dos formas de la hipótesis de interacción: la forma "fuerte" y la forma "débil". La forma "fuerte" es la posición en que la propia interacción contribuye al desarrollo del lenguaje. La forma "débil" que es cuando la interacción es simplemente una forma en que los alumnos encuentran oportunidades de aprendizaje, sean o no de un uso productivo."

\section{Enseñanza del idioma Inglés a temprana edad}

Actualmente existen innumerables posturas sobre cuál es la edad óptima para la enseñanza- aprendizaje del idioma extranjero inglés, y a continuación se presentan las dos posturas ambivalentes más influyentes. Eric. H. Lenneberg, pionero de los estudios sobre el componente biológico del lenguaje humano, propuso un período crítico para la adquisición del lenguaje que finalizaba junto con la pubertad debido a la terminación de la lateralización hemisférica y de la plasticidad cerebral. Según explica Lenneberg (1967:125-178), desde el nacimiento, el hemisferio cerebral dominante se va especializando progresivamente en el lenguaje hasta que, en la pubertad, todas las funciones lingüísticas se concentran en esa parte del cerebro. De acuerdo a Elaine E. Tarone (1976:233-234) solo nos limitamos a una "atrofia" - que se llama "phonological fossilization" - a los hábitos fisiológicos y sugiere que quizás la "flexibilidad" del cerebro disminuya con la edad y que afecte a la pronunciación de la segunda lengua más que a su sintaxis o su semántica. De esta teoría de condicionamiento biológico se infiere que el momento óptimo para aprender una lengua es la niñez, dada la plasticidad cerebral y la falta de especialización cortical que caracterizan esta etapa de la vida: a medida que maduramos y la organización del cerebro se hace más especializada, nuestra capacidad para aprender un idioma tiende a decrecer.

\section{El idioma inglés en aula como facilitador del aprendizaje.}

El docente es la principal fuente de exposición del estudiantado al idioma inglés y las instrucciones y órdenes son los medios naturales de comunicación en inglés en la sala de clases, por lo tanto, es de primera importancia que el docente realice su clase en este idioma. (Planes Y programas de Inglés para el NB5, 2004:10). El uso del idioma Inglés en la sala de clases es primordial para que el estudiante pueda tener la primera aproximación a la lengua extranjera y como lo menciona Scrivener, (2005) “Los profesores son la fuente principal de entrada del idioma comprensible 
para los alumnos, el tiempo que el profesor ocupa en hablar juega una parte importante en la adquisición del lenguaje".

\section{Actividades motivadoras en el aprendizaje.}

El término "motivación" por sí sólo es bastante difícil de definir. Es más útil pensar en el término de "alumno motivado": un alumno que esté dispuesto o incluso dispuesto a invertir esfuerzo en actividades de aprendizaje y progresar, es un aprendiz motivado y hace que la enseñanza y el aprendizaje sea inconmensurablemente más fácil y más agradable, así como más productivo; de ahí la importancia del tema para los docentes. Girard (1977) enfatizó que una parte importante del rol del profesor es motivar a sus estudiantes. Sin embargo, la función del maestro es vista principalmente como aquel proveedor de materiales y condiciones para el aprendizaje, mientras que el estudiante asume la responsabilidad de su motivación y del rendimiento.

\section{Importancia del inglés como segunda lengua.}

Crystal (2003) menciona que la necesidad de una lengua global es particularmente apreciada por la academia internacional y las comunidades de negocios, y es aquí que la adopción de una sola lingua franca es requerida en salas de cátedras y salas de clases. Es entonces, más que una herramienta, es una necesidad del ser humano el poder comunicarse, ya que más allá de la tecnología existente es de vital importancia una lengua en la cual todo el mundo pueda relacionarse, siendo enfáticos en que su importancia no radica sólo en el proceso de comunicación sino que en la habilidad y en las capacidades que entrega a quienes lo estudian. Y como lo menciona Krashen (2007) la importancia de aprender una segunda lengua no está meramente limitada a la habilidad de comunicarse con personas que hablan lenguajes diferentes, sino en la capacidad de los estudiantes de pensamientos divergentes, mayor creatividad, y desarrollo cognitivo más elevado comparado con niños que son monolingües.

Es así como el inglés, se transforma en la lingua franca, la cual es necesaria mundialmente y no se limita sólo a transacciones comerciales sino que mucho más hoy en día cuando nos enfrentamos directo a su enseñanza, la cual evidentemente aporta mayores capacidades y habilidades para nuestros estudiantes.

\section{Comprensión Auditiva}

Otra habilidad del idioma inglés es la auditiva. De acuerdo a Downs (2008) "la palabra audición es definida como el hacer $u$ esfuerzo por oír algo: poner atención o hacer caso a lo oído. Es diferente del escuchar simplemente, lo que entra a ser 
el proceso psicológico del oído absorbiendo ondas de sonido y transfiriéndolas entre las rutas neurales a las distintas partes del cerebro. El escuchar es necesario para la audición, pero ésta última es mucho más que simplemente procesar el sonido. Alguien puede escuchar muy bien, pero no es un muy buen oyente." (p. 1). La comprensión auditiva involucra una serie de procesos complejos que van desde la percepción y procesamiento de una señal acústica variable (Liberman, 1967) hasta la construcción y aplicación de esta representación mental integrada de los eventos descritos (Pisoni, 1996). Es decir, la comprensión requiere de conexiones entre los eventos y la representación existente en la memoria.

\section{Audición: Reproducción de información específica en textos orales.}

Según Wipf (1984) Audición y comprensión auditiva puede definirse como un proceso mental invisible lo que lo hace difícil de describir. Las personas que escuchan deben discriminar entre los diferentes sonidos, comprender el vocabulario y las estructuras gramaticales. Interpretar el énfasis y la intención y retener e interpretar todo lo que está dentro del contexto inmediato, como un contexto socio- cultural más amplio (p, 135). Brown (1984) explica que la comprensión auditiva es el modo de aprendizaje más eficaz, hasta por lo menos el sexto grado de la educación primaria y que alrededor del $60 \%$ de tiempo lectivo de un estudiante se invierte en escuchar $(p, 39)$. Por otro lado es necesario tomar en cuenta la capacidad de atención que los alumnos tienen al momento de escuchar un texto auditivo. En ese sentido Lapuente F, et al (2009) definen el concepto de atención como la habilidad del sujeto para atender a estímulos específicos sin ser distraído por estímulos internos o ambientales.

\section{Comprensión Lectora}

Dentro de las habilidades del idioma del inglés se encuentra la lectura la que, según Romero (1985), se define y caracteriza como un concepto que tiene dos posibles enfoques. Una definición enfatiza los procesos mentales involucrados en la decodificación, mientras que otra definición define los valores que pueden ser derivados del proceso. A pesar de las variaciones de las definiciones del acto de la lectura, esta envuelve el uso de un código que tiene que ser interpretado para el significado. Las definiciones de lectura, pueden clasificarse en 2 categorías. Primero están los [teóricos] que ven la lectura principalmente como un proceso de decodificación, una decodificación del código visual. En una segunda mirada, la lectura para el significado es enfatizada desde las primeras etapas de la instrucción. En esta visión, la lectura como un proceso de comprensión es destacada. 


\section{Comprensión de información específica de textos escritos.}

Para la comprensión de información específica de textos escritos se deben tener en cuenta diversos aspectos, tales como el nivel gramatical morfológico, sintáctico y semántico logrado por los estudiantes; y por medio de la unión de estos aspectos del lenguaje y la comunicación se logra la comprensión. Es por esto que, de acuerdo con Paulo Freire (1996), “Lenguaje y realidad se vinculan dinámicamente. La comprensión del texto al ser alcanzada por su lectura crítica, implica la percepción de relaciones entre el texto y el contexto." Un estudiante de una lengua extranjera quien dice, 'Yo puedo leer las palabras pero no entiendo lo que significan' en este sentido, no es necesariamente leer. Él o ella están meramente decodificando-traduciendo símbolos escritos en su sonido correspondiente." Es mediante estos factores que los establecimientos educacionales buscan lograr e impulsar la comprensión y obtención de información específica de textos escritos.

\section{Producción escrita}

En relación a las habilidades de producción del idioma inglés, en la escritura se resalta la forma retórica y lingüística del texto mismo. Según Nunan (1991), el lenguaje escrito se considera más complejo que el lenguaje oral. Dicha complejidad viene determinada por la densidad léxica (lexical density) (Tribble, 1996:18), es decir, por el número de palabras léxicas o de contenido que se utilizan en el lenguaje escrito. Es por esto que es importante desde la base del aprendizaje de un nuevo idioma, el poder sentar las bases de la escritura, ya que a través de esta, tal como en la producción oral, se pueden concretar los conocimientos adquiridos de la nueva lengua, y comenzar un proceso que no sólo involucra a quién produce el texto escrito, sino también la de poder comunicarse con otros individuos. La escritura es una parte importante del proceso de comunicación no sólo como medio de comunicación sino también como fuente de poder, como necesidad social y como una forma de obtener conocimiento y de resolver problemas (Lindemann, 1987).

\section{Habilidad de producción: Elaboración de textos escritos mediante información específica.}

Para la elaboración de un texto escrito, se considera que los estudiantes deben manejar un adecuado vocabulario, ortografía, estructura del texto en general de frases y oraciones simples; que se encuentran consideradas en su nivel de idioma inglés. Es por esto que según el estudio sobre la relación existente entre competencia en la L2 y producción escrita, realizado por Cumming (1994), se concluye que el factor tiempo empleado en el aprendizaje de la L2 es un factor 
determinante en la competencia adquirida. Es por ello que los colegios buscan en los estudiantes la interiorización de estos aspectos para la elaboración de un texto; junto a su exposición y práctica del idioma de forma escrita a temprana edad.

\section{OBJETIVOS}

El objetivo de esta investigación es develar el impacto de la enseñanza del inglés a temprana edad en estudiantes de cuatro establecimientos municipales de la ciudad de Temuco. De ello, se desprenden los siguientes objetivos específicos: (1) develar el grado de exposición al idioma inglés que presentan los estudiantes de octavo año de enseñanza básica de cuatro establecimientos municipales de la ciudad de Temuco, durante el proceso de aprendizaje del inglés como segunda lengua. (2) medir el nivel de comprensión y producción en el idioma extranjero inglés de estudiantes de octavo año de enseñanza básica de cuatro establecimientos municipales de la ciudad de Temuco, considerando dos que han iniciado sus clases desde quinto y dos desde primero básico. (3) determinar el efecto de la enseñanza del inglés a temprana edad en estudiantes de octavo año de enseñanza básica de cuatro establecimientos municipales de la ciudad de Temuco.

\section{METODOLOGÍA}

Esta investigación tiene la intención de develar la etapa apropiada para la adquisición del inglés como segunda lengua. Se establece realizar un análisis cualitativo de los datos obtenidos durante la investigación. Así como lo plantea Rodríguez Gómez, los investigadores cualitativos estudian la realidad en su contexto natural, tal como sucede, intentando sacar sentido de o interpretar los fenómenos de acuerdo con los significados que tienen para las personas implicadas. La investigación implica la utilización y recogida de una gran variedad de materiales que describen la rutina y las situaciones problemáticas y los significados en la vida de personas. (Rodríguez Gómez y otros, 1996:72). Los instrumentos utilizados son el grupo focal y la prueba de medición del idioma inglés, los cuales sirven para la observación y posterior análisis cualitativo de la información recopilada. Además, se debe aclarar en este punto de la metodología que el hecho de utilizar una prueba de medición tiene como propósito medir el conocimiento de los sujetos en observación arrojando resultados que son más bien cuantitativos. Sin embargo, el análisis de aquellos resultados es netamente cualitativo, pues se busca analizar de forma descriptiva las semejanzas y/o diferencias entre los dos grupos en cuestión (alumnos que aprenden inglés desde quinto año de enseñanza básica y los alumnos que aprenden desde primer año de enseñanza básica). 
Para la presente investigación, el grupo de participantes fue definido de un conjunto de estudiantes de octavo año de enseñanza básica pertenecientes a cuatro establecimientos diferentes de la ciudad de Temuco. Se consideraron dos grupos de estudiantes que han aprendido a temprana edad el idioma del inglés específicamente desde primer año de enseñanza básica y dos grupos de estudiantes que han aprendido el idioma del inglés desde el segundo ciclo de enseñanza básica, específicamente quinto año de enseñanza básica. Finalmente, al considerar los criterios de inclusión y exclusión, se destinó esta investigación a establecimientos públicos municipales que fueran representativos de la realidad chilena y las políticas educativas establecidas, excluyendo los establecimientos particulares pagados y subvencionados por el Estado debido a que su alcance en cuanto a la enseñanza del idioma inglés se aborda en su mayoría desde el primer año de enseñanza básica, lo cual invalidaría esta investigación.

Los instrumentos que se implementaron en esta investigación son: una entrevista semiestructurada aplicada a un grupo focal y una prueba de medición Key English Test (KET Test). El grupo focal consistió de una discusión basada en seis preguntas con cinco estudiantes de octavo año de enseñanza básica de cada establecimiento por separado, considerando una duración de quince minutos como máximo. Aquellos fueron seleccionados mediante el azar, y la categoría principal del grupo focal es "el grado de exposición del idioma inglés que poseen los estudiantes. Considerando seis subcategorías, la primera es "Exposición a material autentico.", la segunda "Exposición al lenguaje.", la tercera "Enseñanza del inglés a temprana edad.", la cuarta "Clase en inglés: facilitador del aprendizaje", la quinta "Clase de inglés: motivadora para los estudiantes", y la última es la "Importancia atribuida a la asignatura de inglés.". Este instrumento tiene como objetivo develar el grado de exposición al idioma del inglés que poseen los estudiantes. En cuanto a la prueba de medición, el Key English Test es una prueba internacional certificada por Cambridge University Press, utilizada también por el gobierno de Chile en la prueba SIMCE de inglés para enseñanza básica. Esta prueba se aplicó a los estudiantes de octavo año de enseñanza básica, incluyendo a aquellos estudiantes que han aprendido el idioma desde primer año de enseñanza básica y otros que han aprendido desde quinto año de enseñanza básica. Los ítems que se usaron son una adaptación que consta de tres partes: comprensión auditiva, comprensión lectora y producción escrita. Cabe señalar que si bien es un instrumento de enfoque cuantitativo, el análisis y los resultados serán definidos bajo un enfoque netamente cualitativo. La prueba de medición tiene como duración setenta minutos y como categorías principales la "comprensión auditiva", "comprensión lectora" y "producción escrita". De estas categorías se desprenden tres subcategorías, "audición", (audición de seis textos auditivos cortos de información específica), 
"lectura" (cinco conversaciones breves de información específica), y "habilidad de producción" (redacción de una postal; siguiendo instrucciones y requerimientos básicos). Las categorías analizadas corresponden al grado de exposición al idioma Inglés. De ésta, se desprenden seis subcategorías: exposición a material autentico; exposición al idioma inglés; enseñanza del inglés a temprana edad; uso del inglés como facilitador del aprendizaje; actividades motivadores en el aprendizaje e importancia del inglés como segunda lengua. En la prueba KET se encuentran tres categorías: comprensión auditiva, comprensión lectora y producción escrita. De ésta se desprenden las microcategorías denominadas: reproducción de información específica en textos orales, comprensión de información específica en textos escritos breves y elaboración de textos escritos mediante información específica respectivamente.

\section{ANALISIS Y RESULTADOS}

Con el propósito de realizar un análisis más profundo, se decidió utilizar dos tipos de análisis distintos: análisis de datos de tipo descriptivo utilizando el estudio de grupo focal y el análisis de datos mediante la aplicación de una prueba internacional de medición del nivel de inglés KET.

\subsection{GRUPO FOCAL}

El tipo de análisis utilizado en este estudio es el de análisis de contenido de tipo descriptivo, el cual es útil al momento de realizar grupos focales con los participantes.

\subsubsection{Grado de exposición al idioma Inglés.}

Esta categoría se enfoca en el grado de exposición que los estudiantes actualmente poseen, y devela las principales nociones en relación a cuán expuestos estos se encuentran a la lengua extranjera y cómo determinan la utilidad de esta en su propio proceso de enseñanza- aprendizaje. De esta categoría, se desprenden seis subcategorías: exposición a material auténtico; exposición al lenguaje; enseñanza del inglés a temprana edad; clase en Inglés: facilitador del aprendizaje; clase de inglés: motivadora para los estudiantes e importancia atribuida a la asignatura de Inglés respectivamente.

\subsubsection{Exposición a material autentico.}

Geddes y White (1978) recomiendan el uso del material auténtico tanto como sea posible en la enseñanza. Como ellos mismos han notado, este movimiento hacia 
la autenticidad en la enseñanza del lenguaje refleja un incremento en interés hacia las funciones comunicativas en los últimos años. En los siguientes extractos es posible notar la importancia que se le da al uso del material autentico por parte de los participantes.

"En mi caso sería todos los días, música, videos, películas puede ser día por medio, pero música en inglés siempre, todos los días".

"Eh diariamente casi todo el rato". "Desde que llego del colegio empiezo a jugar, ver películas y siempre están en inglés, sobre todo las películas y video juegos, mucho, tres horas, cuatro horas al día".

Los participantes, en general, consideraron la exposición al Inglés con un alto grado de utilidad en función de su propio aprendizaje o como un método de entretenimiento, asociando el lenguaje a sus gustos e intereses, destacando que 10 de 20 estudiantes atribuyeron sus preferencias por la música y videos, 4 de 20 asocian su afición por las películas y en igual cantidad por los videos juegos. Cabe señalar que sólo 2 de 20 estudiantes indicaron no tener proximidad con el inglés.

\subsubsection{Exposición al idioma Inglés}

Rogers y Medley (1988) se refieren a material auténtico como ejemplos de lenguaje, ya sea oral o escrito, que refleja una naturaleza de forma y una propiedad de contexto cultural y situacional que podría ser encontrado en el lenguaje como es usado por un hablante nativo. Es posible notar mediante los siguientes extractos la importancia que los estudiantes le otorgan a las interacciones con hablantes nativos del idioma extranjero Inglés:

“....Si, yo también, es que mi abuelita ha ido a Estados Unidos varias veces, y sabe hablar bien inglés. Entonces eh ella tiene amigos allá en Estados Unidos, y trajo a uno de sus amigos acá para que conociera Chile y todo eso".

"....yo si profesora, es que mi hermana trajo a su amiga a la casa una vez, y la amiga venía con una niña que era como inglesa o algo así, entonces tomaron once en la casa y hablaba español y de repente la llamaron por teléfono y empezó a hablar en Inglés y entonces yo le dije ¡Hello! Y me respondió así, hablaba super bacán, le pregunte por la Demi Lovato, por unas canciones que me gustaban y me las tradujo". "si igual harto, porque uno siempre escucha en las películas como hablan, pero ahora era como más difícil, porque usaba palabras como complicadas que yo nunca había escuchado".

Los estudiantes reconocen haber sido expuestos al lenguaje oral autentico, destacándose 14 de 20 estudiantes quienes han sido sometidos a alguna situación 
comunicativa que incluya conversaciones o simples diálogos con hablantes nativos del idioma extranjero Inglés. En aquella oportunidad, destacó el hecho de no entender debido a la complejidad del idioma al que se encontraban expuestos. Sin embargo, la mayoría considera esta oportunidad como una situación motivante.

\subsubsection{Enseñanza del Inglés a temprana edad:}

La enseñanza del idioma inglés a temprana edad se entenderá como el proceso de enseñanza aprendizaje que se extenderá durante los primeros años de vida. De acuerdo a Elaine E. Tarone (1976: 233-234) solo nos limitamos a una "atrofia" que se llama "phonological fossilization" - a los hábitos fisiológicos y sugiere que quizás la "flexibilidad" del cerebro disminuya con la edad y que afecte a la pronunciación de la segunda lengua más que a su sintaxis o su semántica. Lo expuesto anteriormente se evidencia a través de los siguientes relatos:

"Es que es importante porque cuando uno piensa ir a otro país obviamente, va a tener toda la base. Siempre se ha sabido que los primeros cinco años es cuando uno ya empieza a estructurar la (...) del cerebro, entonces si uno va con el inglés desde esos años, entonces uno ya tiene la base".

"Eh, yo pienso que practicar inglés desde pequeño sería muy importante, ya que se tiene más facilidad en aprenderse el lenguaje" "...como el español se puede aprender prácticamente se puede aprender todo lo que más se pueda, mientras que crece el cerebro se aprende eso se aprenden todos esos idiomas".

Es posible mencionar que los estudiantes atribuyeron a la enseñanza del inglés una importancia considerable en función de su aprendizaje y el período de edad, destacando que el total de la muestra atribuye una preocupación por aprender este idioma lo más temprano posible. Además, reconocieron su funcionalidad no sólo como una estrategia de comunicación, sino como una necesidad de adquirir mejores oportunidades y facilidades a nivel país generando funcionalidad y considerándolo significativo para ellos.

\section{$\underline{4.1 .5}$ Uso del inglés en aula como facilitador del aprendizaje}

El uso del idioma Inglés en la sala de clases es primordial para que el estudiante pueda tener la primera aproximación a la lengua extranjera y como lo menciona Scrivener, (2005) "Los profesores son la fuente principal de entrada del idioma comprensible para los alumnos, el tiempo que el profesor ocupa en hablar juega una parte importante en la adquisición del lenguaje". En los siguientes extractos se evidencia el uso del inglés como un factor crucial en el proceso de enseñanzaaprendizaje de los participantes: 
"Yo creo que depende porque si es que no sabe inglés no entendería nada y se haría difícil, pero si tiene buena base, demás que sería más fácil entender si hablan toda la clase en inglés".

"Lo mismo (risas), no es que no puede dar la clase en Inglés si uno no sabe mucho, no se maneja en eso y uno no aprende nada al final'. Claro, porque para uno es fome escuchar todo en Inglés es mejor cincuenta y cincuenta como decía la Florencia".

Dentro de esta subcategoría, fue posible establecer que mayoritariamente los estudiantes mencionaron que una clase impartida bajo un uso completo de esta lengua provoca desmotivación y carencia del aprendizaje al momento de ser enseñada, considerando principalmente que la base adquirida es un factor crucial que determina la comprensión y reproducción del lenguaje oral.

\subsubsection{Actividades motivadoras en el aprendizaje.}

Girard (1977) enfatizó que una parte importante del rol del profesor es motivar a sus estudiantes. Asimismo, los escolares sólo pueden aprender bien un idioma extranjero si se encuentra una manera de activar y estimular su deseo de invertir esfuerzos en la actividad de aprendizaje. Esto se esclarece en los siguientes extractos del grupo focal:

“...Para mí son más motivadoras las canciones y lectura. Lo otro igual sería, que el año pasado nos hacían juegos con las canciones, eso era entretenido y siento que aprendia mejor, es que aprendo cuando me entretengo. "...Canciones y dinámicas podría ser. A mí por lo menos lo último que me motivó fue lo último que hicimos en inglés que nosotros tuvimos que investigar sobre un país y toda la información de ese país tuvimos que hacerlo todo en inglés. Y poner un video, de un cantante o una banda o lo que sea de ese país, y disertarlo. Más que nada eso es lo que más me motivó".

Los estudiantes señalan como situaciones de aprendizaje motivantes aquellas que están directamente relacionadas a sus gustos personales y preferencias, tales como canciones, traducción de éstas, dinámicas, videos y disertaciones. Sin embargo 18 de 20 estudiantes menciona que en aula existe una inconsistencia de este tipo de actividades, desglosando que sólo 8 de 20 estudiantes ha trabajado en actividades de audición, 3 de 20 en traducir canciones o realizar disertaciones, 4 de 20 en dinámicas grupales o competencias y finalmente 5 de 20 estudiantes nunca se ha visto envuelto dentro de este tipo de actividades.

\section{$\underline{4.1 .7 ~ I m p o r t a n c i a ~ d e l ~ i n g l e ́ s ~ c o m o ~ s e g u n d a ~ l e n g u a ~}$}


Crystal (2003) menciona que la necesidad de una lengua global es particularmente apreciada por la academia internacional y las comunidades de negocios, y es aquí que la adopción de una sola lingua franca es requerida en salas de cátedras y salas de clases, igual que en miles de contactos individuales hechos diariamente en todas partes del mundo. En los siguientes extractos se puede clarificar lo anteriormente expuesto:

“....y aparte que uno necesita el Inglés para todo, no sólo para estudiar porque si uno se da cuenta ahora todo viene en Inglés, cuando uno compra en las etiquetas todo está escrito en inglés, entonces más encima si uno quiere estudiar una carrera universitaria es necesario para saber más".

“...Yo digo que es importante que enseñan cualquier cosa en inglés, por ejemplo si uno estudia medicina igual le van a enseñar inglés, en realidad en todas las carreras hay inglés parece. Además si yo quiero estudiar, por ejemplo, alguna ingeniería, igual voy a necesitar inglés para salir a otros países a hacer negocios o cosas así. Por eso creo que es importante igual".

Los estudiantes asignaron importancia a la asignatura de inglés, destacando que 19 de 20 estudiantes concordaron con esta aseveración. Los estudiantes reconocieron su utilidad en un mundo globalizado y percibieron su importancia de manera trascendente incluyendo aparte de su función comunicativa y meramente educacional mejores oportunidades de trabajo, estadías en el extranjero, becas, asociaciones y transacciones de negocios. Además, atribuyeron su importancia como parte del Curriculum nacional necesario para obtener mejores oportunidades en distintas etapas de enseñanza, considerando prioritariamente carreras universitarias.

\subsection{PRUEBA INTERNACIONAL DE MEDICIÓN KET}

\subsubsection{Comprensión Auditiva:}

Según Wipf (1984), audición o comprensión auditiva puede definirse como un proceso mental invisible lo que lo hace difícil de describir. Los textos de audición seleccionados corresponden a una adaptación y selección de tres distintos tipos de audición nivel KET. Los tres corresponden a textos auditivos con ejercicios de información específica. En el primero, son cinco conversaciones breves distintas, en las cuales el estudiante debe elegir la respuesta correcta. La segunda es un solo texto auditivo con cinco preguntas de selección múltiple acerca de una mujer hablando de sus vacaciones. Finalmente, el tercero corresponde a un texto auditivo relacionado a una conversación telefónica de una mujer preguntando cosas acerca 
de excursiones en un castillo. La subcategoría de la parte de audición del test corresponde a la audición y reproducción de información específica en textos orales.

\subsubsection{Audición: Reproducción de información específica en textos orales.}

La comprensión auditiva involucra una serie de procesos complejos que van desde la percepción y procesamiento de una señal acústica variable (Liberman, 1967) hasta la construcción y aplicación de esta representación mental integrada de los eventos descritos. Dentro de esta categoría, en términos generales se puede señalar que menos de la mitad del total de los alumnos pudieron obtener información general y especifica de los audios aplicados. En el siguiente dato se evidencia lo anteriormente señalado:

\section{Pregunta 1: What time will the party start?}

Solo 26 de 80 alumnos respondieron B) 9:30 hrs, que era la respuesta correcta.

Si se menciona el nivel de aprendizaje y se toma en cuenta los resultados obtenidos, se puede afirmar que los estudiantes están bajo el promedio o nivel de aprendizaje de inglés como segunda lengua en el cual ellos debieran estar, de acuerdo a los estándares con los cuales mide la prueba SIMCE de inglés.

Brown (1984) explica que la comprensión auditiva es el modo de aprendizaje más eficaz, hasta por lo menos el sexto grado de la educación primaria y que alrededor del $60 \%$ de tiempo lectivo de un estudiante se invierte en escuchar (p, 39). Se puede decir entonces que, debido a que los alumnos ya no se encuentran en esa etapa, su capacidad de comprensión auditiva no es lo suficientemente eficaz como para poder realizar ejercicios de información especifica que de por sí requieren mayor atención. Lapuente F, et al (2009) define el concepto de atención como la habilidad del sujeto para atender a estímulos específicos sin ser distraído por estímulos internos o ambientales. Este concepto estaría refiriéndose a la capacidad que presenta una persona para concentrarse en una cierta estimulación aferente (externa), programas motores, memorias o representaciones internas (mentales).

Es posible observar los datos obtenidos:

D 1: Los resultados más altos se dan en las preguntas 5 y uno las que corresponden a vocabulario de "como ver la hora" y "días de la semana".

Se puede inferir del primer texto auditivo que los estudiantes no manejan vocabulario básico relacionado al clima, frutas y medios de transporte, pues los resultados indican que menos de la mitad no entendió las conversaciones relacionadas a ese vocabulario. 
D 2: Los resultados de la parte dos correspondiente a un texto auditivo relacionado al tema de los: "deportes", "dias de la semana". Dan resultados en los cuales denotan que hubo mejor comprensión en la pregunta 10 correspondiente al dia viernes (friday).

Se puede deducir de la segunda audición que el nivel de comprensión auditiva del idioma extranjero que los alumnos tienen es muy bajo, lo que se puede deber a la falta de exposición a material autentico en ingles. Llámense películas, música, videos, etc.

$D$ 3: Con respecto a la parte tres donde los alumnos debian escribir con sus propias letras las respuestas los resultados fueron negativos en general pues solo un máximo de 25 de un total 80 logro responder correctamente las preguntas 11, y 12 y el resto de preguntas de este item fue de menor resultado.

Se puede inferir del tercer texto auditivo que los alumnos pueden captar información general, pero no especifica. Además de no poder escribir con sus propias palabras lo que escuchan y/o logran captar y comprender.

\subsubsection{Comprensión Lectora:}

Por medio de esta categoría, queda en manifiesto el nivel de comprensión de información que logran satisfactoriamente los estudiantes, utilizando textos escritos breves. A partir de esta categoría se desprende la subcategoría: Lectura; la cual a su vez, contiene la micro categoría: Comprensión de información explicita en textos escritos breves.

\subsubsection{Lectura: Comprensión de información específica de textos escritos.}

Para la comprensión de lectura, podemos acotar dos puntos de vista que se complementan en la importancia de la misma. D e acuerdo con el estudio acerca de la importancia de leer de Paulo Freire (1996), en el cual se indica que lenguaje y realidad poseen una relación intrínseca, en la cual la relación entre el texto y el contexto son fundamentales para lograr la comprensión lectora. Según los resultados, los estudiantes de escuelas públicas municipales (públicas) no logran superar la exigencia mínima del $70 \%$ de respuestas acertadas por cada alternativa. Esto quiere decir que esta muestra de estudiantes no posee, en su gran mayoría, la habilidad de comprensión lectora adecuada a su nivel para extraer información específica de forma acertada. Se puede deducir también que la falta de comprensión se debe a la no asimilación adecuada de los estudiantes con el contexto de ambas lecturas aplicadas. 
A continuación observamos los resultados primer texto de lectura; desde la pregunta 16 a la 20 y junto a ella la cantidad de respuestas correctas de los estudiantes:

Tabla $N^{\circ} 1-$ Resultados Primer texto de lectura.

\begin{tabular}{|c|c|c|c|c|c|c|c|}
\hline $\begin{array}{l}N^{o} \\
\text { Pregunta }\end{array}$ & $\begin{array}{l}\text { Resp } \\
\text { Correctas }\end{array}$ & $\begin{array}{l}N^{o} \\
\text { Pregunta }\end{array}$ & $\begin{array}{l}\text { Resp. } \\
\text { Correctas }\end{array}$ & $\begin{array}{l}N^{o} \\
\text { Pregunta }\end{array}$ & $\begin{array}{l}\text { Resp. } \\
\text { Correctas }\end{array}$ & $\begin{array}{l}N^{\circ} \\
\text { Pregunta }\end{array}$ & $\begin{array}{l}\text { Resp. } \\
\text { Correctas }\end{array}$ \\
\hline 16 & 25 & 17 & 45 & 18 & 33 & 19 & 29 \\
\hline $\begin{array}{l}N^{o} \\
\text { Pregunta }\end{array}$ & $\begin{array}{l}\text { Respuesto } \\
\text { Correctas }\end{array}$ & & & & & & \\
\hline 20 & 25 & & & & & & \\
\hline
\end{tabular}

Por último, podemos observar los resultados obtenidos en el segundo texto de lectura desde la pregunta 21 a la 27 y junto a ella la cantidad de respuestas correctas de los estudiantes:

Tabla $\mathbf{N}^{\circ}$ 2- Resultados segundo texto de lectura

\begin{tabular}{|c|c|l|l|l|l|l|l|}
\hline$N^{\circ}$ & $\begin{array}{l}\text { Resp. } \\
\text { Pregunta }\end{array}$ & $N^{\circ}$ & $\begin{array}{l}\text { Resp. } \\
\text { Correctas }\end{array}$ & $N^{\circ}$ & $\begin{array}{l}\text { Resp. } \\
\text { Pregunta }\end{array}$ & $N^{\circ}$ & $\begin{array}{l}\text { Resp. } \\
\text { Correctas }\end{array}$ \\
\hline 21 & 33 & 22 & 31 & $\mathbf{2 3}$ & 27 & $\mathbf{2 4}$ & 27 \\
\hline
\end{tabular}

\begin{tabular}{|c|c|l|l|l|l|}
\hline$N^{\circ}$ & $\begin{array}{l}\text { Respuestas } \\
\text { correctas }\end{array}$ & $\begin{array}{l}N^{\circ} \\
\text { Pregunta }\end{array}$ & $\begin{array}{l}\text { Respuestas } \\
\text { correctas }\end{array}$ & $\begin{array}{l}N^{\circ} \\
\text { pregunta }\end{array}$ & $\begin{array}{l}\text { Respuestas } \\
\text { correctas }\end{array}$ \\
\hline 25 & 38 & 26 & 24 & 27 & 30 \\
\hline
\end{tabular}

De acuerdo a los resultados analizados acerca del primer texto de lectura que consistía en preguntas de selección múltiple, la mayoría de los estudiantes no obtuvieron una cantidad óptima de respuestas correctas, destacando solamente una alternativa en la que obtuvieron más de la mitad de aciertos. En el segundo texto, los resultados fueron similares en cuanto a respuestas acertadas; que entre ambos logran menos de la mitad de estudiantes con respuestas correctas.

\subsubsection{Producción Escrita:}

Esta categoría engloba la habilidad de producción escrita de un texto breve que puedan desarrollar los estudiantes. Esta consiste en la producción de un texto de 25 a 35 palabras, en la que se debe incluir tres datos de información específica 
requerida. Para la producción de este texto, se considera el nivel que tienen los estudiantes, el cual dejará de manifiesto sus logros de producción alcanzados. De esta categoría se desprende la subcategoría: Habilidad de producción, la que a su vez contiene la micro categoría: Elaboración de textos escritos mediante información específica.

4.2.3.1 Habilidad de producción: Elaboración de textos escritos mediante información específica.

La escritura es otra parte importante del proceso de comunicación, ya que a través de esta podemos plasmar el lenguaje hablado y con el nivel logrado de esta habilidad. Mediante la elaboración del texto requerido se busca evidenciar no solo la capacidad de producción de los estudiantes, sino también la forma en que organizan sus ideas en la resolución de problemas y situaciones. Según los datos recogidos, en la pregunta 28 de producción escrita de una tarjeta postal de 25 a 35 palabras. Aquí se detalla primero el número de estudiantes junto a la cantidad de puntos logrados:

\begin{tabular}{|c|c|}
\hline$N^{o}$ de alumnos & Puntajes \\
\hline $\mathbf{1 1}$ & $5 \mathrm{pts}$. \\
\hline $\mathbf{1 1}$ & $2,5 \mathrm{pts}$. \\
\hline $\mathbf{4}$ & $1 \mathrm{pto}$. \\
\hline
\end{tabular}

De acuerdo a los resultados obtenidos, menos de la mitad de los estudiantes lograron producir frases y oraciones simples adecuadas a su nivel del idioma inglés. De un total de 80 alumnos, un máximo de 11 pudieron realizar una actividad de producción escrita con un resultado de 5 puntos (máximo). Tan solo 26 alumnos lograron responder este ítem, obteniendo de entre 1 a 5 puntos.

A través de los resultados obtenidos se pudo evidenciar que no existe implicancia de la edad en relación al aprendizaje de una segunda lengua, ya que los participantes dejaron entrever mediante los resultados de las pruebas de medición puntajes similares e insatisfactorios lo que denota una considerable carencia del aprendizaje en las primeras etapas. Sin embargo, se desprende una leve diferencia de aquellos alumnos que comenzaron sus clases desde primer ciclo de enseñanza básica. Se pueda visualizar también que al considerar el grado de exposición y la edad, existen factores tanto internos como externos, los cuales influyen en cómo el aprendiz comprende y produce el lenguaje. Estos son: la motivación, el grado de exposición por parte del profesor, recursos para el aprendizaje y trasfondo cultural. Cabe señalar de esta forma que el grado de exposición es uno de los factores a considerar dentro del aprendizaje, ya que tanto los recursos utilizados 
(material autentico, canciones, interacción con hablante nativos y actividades motivadoras) como los profesores son la fuente principal para que el aprendiz tenga su primer acercamiento con la lengua extranjera. Es de esta forma que se torna una necesidad el reforzar el aprendizaje con recursos y materiales que motiven a los educandos, para asegurar que los estudiantes en etapas primarias alcancen el nivel de inglés esperado.

\section{DISCUSIÓN}

Considerando los resultados obtenidos, se puede concluir que no existe mayor impacto en cuanto a la enseñanza del inglés a temprana edad, esto tomando en cuenta los resultados obtenidos en la prueba de medición KET. A pesar que la mitad de los estudiantes evaluados tuvo acceso a la enseñanza del idioma inglés desde primer año de enseñanza básica, tan solo el 50\% de ellos logró responder de manera satisfactoria. Esto se debe posiblemente a diversos factores externos que influyen en su aprendizaje, tales como: carencias e inequidades de tipo económicas, el contexto cultural y social, recursos y materiales implementados por los profesores y establecimientos. Además, en el proceso de aprendizaje del idioma inglés que envuelve a los estudiantes se ve influenciado por factores como la exposición a material autentico, la exposición y la enseñanza del idioma inglés a temprana edad, el uso del inglés en el aula como facilitador del aprendizaje, actividades motivadoras en el aprendizaje e importancia del inglés como segunda lengua. Se confirma, a través de los datos contabilizados y analizados, que el hecho de estar expuestos al idioma tanto de manera formal como informal les ha beneficiado e influenciado positivamente a los estudiantes en la familiarización con el idioma inglés y en la identificación con actividades que ellos disfrutan. Se puede señalar de igual manera que en cuanto al grado de exposición a material autentico, los alumnos no tienen un nivel de exposición suficiente al comprender y reproducir el segundo idioma de manera efectiva y significativa. Esto, a pesar que ellos escuchan música en inglés, ven películas e interactúan con regularidad con hablantes nativos. Los resultados de la prueba indican que el nivel de comprensión auditiva, lectora, y de producción de textos escritos es deficiente. Cabe destacar que solo una mínima cantidad de los estudiantes que comienzan a tener exposición al inglés de manera formal desde primer ciclo de enseñanza básica logran un mayor nivel de comprensión y producción del idioma, comparándolos con a aquellos estudiantes que comenzaron desde segundo ciclo. Esto gracias al uso y la familiarización de estructuras gramaticales aprendidas a temprana edad, específicamente a los seis años para aquellos que comenzaron en primer año de enseñanza básica y diez años para los estudiantes que comenzaron en quinto año de enseñanza básica. 
El efecto provocado por la enseñanza del inglés a temprana edad no fue significativamente relevante en estos estudiantes. Sin embargo, se encontró como principal hallazgo el que los estudiantes que comenzaron a temprana edad con clases de inglés (primer año de enseñanza básica), tienen más facilidad para reconocer vocabulario e identificar la información específica requerida, por sobre sus pares que comenzaron en quinto año de enseñanza básica. Se evidenció que, a pesar que todos los estudiantes empezaron a tener un contacto y exposición con el idioma inglés de manera informal relativamente a la misma edad, los que comenzaron el aprendizaje de manera formal a más temprana edad obtuvieron ligeramente mejores resultados. De esta forma, se percibe que los estudiantes ven el idioma extranjero como un método de entretenimiento relacionado a sus gustos e intereses debido a que declaran tener interés por la música, videos, películas y video juegos. Cabe destacar que los participantes han sido expuestos al lenguaje de forma oral auténtica en su totalidad por medio de hablantes nativos, declarando que este hecho despierta su interés por el idioma. Los estudiantes consideran muy importante aprender el idioma a temprana edad, ya sea para lograr familiarizarse, o bien porque representa una ventaja en la obtención de mejores oportunidades laborales en el futuro. Por otra parte, se devela que los estudiantes prefieren que la clase no sea realizada completamente en el idioma inglés, puesto que consideran que representa una barrera desmotivadora para la comprensión de las actividades dentro del aula, más aún, los estudiantes manifiestan en su gran mayoría que el uso de la lengua materna es sumamente necesaria.

Un hallazgo importante de señalar se refiere a las actividades que los estudiantes señalan como motivadoras. Ellos señalan que las actividades motivadoras son principalmente las relacionadas con sus gustos e intereses personales. De este modo, los estudiantes, en su gran mayoría, consideran el idioma inglés de suma importancia tanto por su función comunicativa como en mejores oportunidades que se pueden obtener gracias a la globalización.

Ante el análisis y la discusión realizada con los datos expuestos, es posible determinar que no se encontraron logros notablemente significativos. Sin embargo, se puede desprender como principal hallazgo el hecho que aquellos estudiantes que comienzan la exposición del idioma inglés a más temprana edad de manera formal logran un mejor aprendizaje, comprensión y motivación con el mismo; esto comparado con aquellos estudiantes que comenzaron a aprender el idioma inglés a una edad más tardía, como lo es en el caso del sistema educativo chileno que por ley exige la enseñanza del idioma inglés como segunda lengua desde quinto año de enseñanza básica. 


\section{REFERENCIAS}

Bonamino, A. Y Franco, C. (2004): Eficacia y Equidad en la Escuela Fundamental Brasilera. Fondo de Investigaciones Educativas - PREAL.

Bourdieu P. (1977) Cultural Reproduction and Social Reproduction. In: Power and Ideology in Education, edited by Jerome Karabel and A. H. Halsey, New York: Oxford University Press.

Bourdieu P. (1986) The forms of capital. In: Richardson, J. G. (Ed.) Handbook of Theory and Research for the Sociology of Education, pp. 241 -258.

Brown D. (1994) Principles of Language Learning and Teaching. Third Edition. Prentice Hall Regents.

Brown, G. and Yule, G, (1983). Teaching in spoken Language. Cambridge: Cambridge University Press.

Case. R. (1989). El desarrollo intelectual. Ediciones Paidós. Barcelona.

Carazo. Z (2010). The use of songs as authentic material: IES Marqués de Comares. Lucena, Córdoba.

Cervini, (2002). Desigualdades en el logro académico y reproducción cultural en Argentina. Un modelo de tres niveles. Revista Mexicana de Investigación Educativa, Vol. 7, N 16, pp. $445-50$

Crystal, D. (2003). English as a Global Language. Cambridge, Inglaterra: Cambridge University Press.

Downs, L. (2008): Listening Skills Training. Baltimore, Estados Unidos: Victor Graphics Inc. Press

Freire, P. (1996) “La importancia del acto de leer" en Enseñar lengua y literatura en el Bachillerato. Textos de Didáctica de la Lengua y de la Literatura, Barcelona, Graó, $\mathrm{n}^{\mathrm{o}} 15$, pagina. 82

Klein, W. (1986). Second Language Acquisition. Cambridge: Cambridge University Press.

Krashen, S. (2007): 'Theory of Second Language Acquisition',

Krashen, S. D., M. A. Long, and R. C. Scarcella. (1979): "Age, Rate and Eventual Attainment in Second Language Acquisition." TESOL QUARTERLY 13 573-582.

Liberman, A. (1967): "Perception of Speech Code" Psychological Review 74, pp. 431-461. 
Lindeman E. (2001): A rethoric for Writing Teachers. Oxford University Press.

MINEDUC, (2004) "Planes y programas de Inglés para el NB5", Ministerio de educación, República de Chile. Santiago.

Muzetti. L. (2000): Escritos de educación. Reseña de: Nogueira, M. A., Catani, A. (Org.). Educación y Sociedad, Año 21, Nº 73, pp. 257 -261.

Nogueira, C., Nogueira, M. (2002): Sociología de educación de Pierre Bourdieu: limites y contribuciones. Educación y Sociedad, Vol.23, No 78, pp. 15- 36.

Nunan, D. (1991): Language teaching methodology: a text for teachers. Prentice Hall International (UK) Ltd.

Piaget J. (1923), "Th e language and thought of the child". Buenos Aires: Guadalupe, 1972.

Pisoni, D. (1996): "Some thoughts on Anormalization in Speech Perception". En

Johnson y J. W. Mullennix (Eds.). Talker variability in speech processing. San Diego: Academic Press.

Raimes, A. (1983) Out of the Woods: Emerging traditions in the Teaching of Writing.TESOL Quarterly.

Romero, A. (1985): Developmental Reading. Manila, Filipinas: Rex Book Store Press.

Resultados de Inglés para Docentes y Directivos SIMCE Inglés. 2010

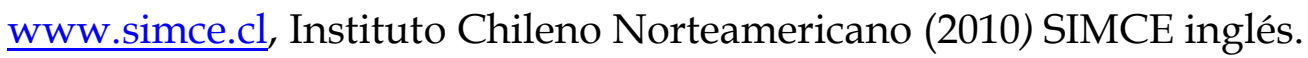

Scrivener, J, and MacMillan (2005), “Learning Teaching. A guidebook for English language teachers". Second Edition.

Tarone, E. (1976): "Some Influences on the Syllable Structure of Interlanguage Phonology", en Interlanguage Phonology: The Acquisition of a Second Language Sound System, IOUP, G. y S. Weinberger, Newbury House Publishers, 1987.

Tribble C. (1996) Writing (Language Teaching: A scheme for Teacher Education). OUP Oxford.

Vygotsky, Lev. (1986), “Thought and Language". Ed. and trans. Alex Kozulin. Cambridge, MA: M.I.T. Press. 
Wipf (1984), Teaching Second Language Listening Comprehension. Foreing Languague Annals. 17, 345- 348.

\section{AUTOR/ES:}

\section{David Quidel Cumilaf:}

Profesor de inglés. Monitor del programa de Inglés Rural perteneciente a la Unidad de Curriculum y Evaluación del Programa Inglés Abre Puertas del Ministerio de Educación.

\section{Juan del Valle Rojas:}

Profesor de inglés e Investigador. Colaborador en publicaciones sobre la enseñanza del idioma inglés, el bilingüismo y trilingüismo en contextos educativos, la educación intercultural y el racismo y la discriminación del pueblo mapuche en Chile.

\section{Lissette Arévalo López:}

Profesora de inglés.

\section{Catherine Nancucheo Chihuaicura:}

Profesora de inglés. Docente en "Colegio Cumbres de Labranza", dependencia municipal, Ciudad de Temuco.

\section{Ramón Ortiz Neira:}

Profesor de inglés. 